\title{
Reflection of Autonomic Regulation Behaviour using Short-Term Cross-Spectral Analysis on RR and QT Intervals
}

\author{
Ping Zhan, Chenxi Li, Hongduoer Liu, Yi Peng \\ Institute of Basic Medical Sciences, Chinese Academy of Medical Sciences \& School of Basic \\ Medicine, Peking Union Medical College, Beijing, China
}

\begin{abstract}
The balance of autonomic nervous system (ANS) is of great significance for regulating normal physiological functions of heart and avoiding risk of cardiac diseases. This project is aimed to reveal the regulation state of ANS by analyzing the linear relationship between $R R$ interval (RRI) and QT interval (QTI). The data for this analysis were provided by the Telemetric and Holter ECG Warehouse (THEW), among which database Normal was selected as normal controls $(n=189)$ and database ESRD as typical subjects of ANS dysfunction with high risk for cardiac arrhythmias and sudden cardiac death( $n=43)$. A causal cross-spectral approach based on the autoregressive model was applied to distinguish the directional effect from RRI to QTI. And the causal crossspectral analysis used here was focused on 2-minutelength series. Firstly, the validity of the short-term indices was verified. Then the relevant indices, such as crossspectral energy in both low frequency (LF) and high frequency (HF) bands, as well as transfer function $G$, were calculated. Inner-comparison for the same index between day and night and inter-comparison between Normal and ESRD were made. Results showed that: 1) For Normal, there were significant differences between daytime and nigh-time results of $G_{L F}, G_{H F}$ and $L F / H F$. However, no significant diurnal variation of the corresponding indices existed in ESRD; 2) $G_{L F-d} / G_{L F-n}$ and $(L F / H F)_{d} /(L F / H F)_{n}$ were significantly smaller in ESRD than that in Normal. The above results suggest the loss of circadian rhythm in ESRD. Furthermore, there is no statistic difference of $G_{H F-d} / G_{H F-n}$ between Normal and $E S R D$. Since HF band mainly reflect the influence of vagal tone, it was revealed that loss of circadian rhythm in ESRD was primarily caused by the overactivity of sympathetic branch. Our study confirmed the feasibility of very short series based analysis and provided an approach for investigating ANS activity.
\end{abstract}

\section{Introduction}

The modulation of autonomic nervous system (ANS), concerning the interaction of sympathetic nerve and vagal nerve, plays a key role in cardiac physiological regulation. The time interval series from ECG are foundational signals, of which RR interval (RRI) and QT interval (QTI) are significant ones. The coupling between RRI and QTI as well as heart rate variability (HRV) and QT variability (QTV) can be changed in different physiological and pathological conditions, reflecting a plenty of information about ANS modulation [1,2].

In addition to the dependence on RRI, QTI is determined by the factors independent of RRI such as the regulation of ANS itself [3,4]. Moreover, the accommodation of QTI to RRI is very complicated, including not only an immediate response to the current RRI but also a chronic response in which the time constant is about 1 2 min [5,6]. Heart period and ventricular repolarization exhibit rhythmical fluctuations and remarkable correlation both in the low frequency $(0.04 \sim 0.15 \mathrm{~Hz}, \mathrm{LF})$ band and high frequency $(0.15 \sim 0.4$ $\mathrm{Hz}, \mathrm{HF}$ ) band $[7,8]$. However, this correlation can be changed by physiological factors (such as day and night alternation) and autonomic neuropathy caused by diseases, such as heart failure, diabetes, myocardial infarction and so on.

In the present study, we applied cross-spectrum based method to analyze possible differences of the short-term indices between normal controls and those with high risk for cardiac arrhythmias and sudden cardiac death. The ANS behavior of these people was characterized by directional correlation analysis of RRI and QTI based on transfer function.

\section{Materials and methods}

\subsection{Data source}

Database Normal (E-HOL-03-0202-003, 9 82 y) from THEW contains 24 h Holter recordings of 202 healthy subjects. The other database is ESRD (E-HOL-12-0051016, >40 y), including $48 \mathrm{~h}$ Holter recordings of 51 end stage renal disease patients with high risk for cardiac arrhythmias and sudden cardiac death. With the exclusion 
of those incomplete records, 189 records from Normal and 43 records from ESRD were finally selected.

For each selected recording, 2 episodes (each lasting 2 h) in resting state were extracted, one in the period of 7:00 20:00(day), the other in 0:00 6:00 (night). RRIs of the selected $2 \mathrm{~h}$ episodes were derived from the annotations in the databases, while QTIs corresponding to the selected RRIs were obtained by using a complex algorithm [9] validated with the records in Physionet QT Database(http://www.physionet.org/physiobank/database/ $\mathrm{qtdb} /$ ). Ectopic beats were removed before further analysis.

\subsection{Methods}

In this study, the cross-spectral analysis based on the AR model was focused on short-term (2 min) indices of RRI and QTI series. The validity of the short-term indices was firstly verified and then the relevant indices (such as transfer function) results were compared between Normal and ESRD in terms of circadian rhythm.

First of all, all the 2h RRI series and QTI series in Normal were intercepted to get 2 min RRI and QTI series. This process was repeatedly done for five times to get five nonoverlapping short-term RRI and QTI data for each recording. Then, cross-spectral analysis based on the autoregressive model (AR model) was performed to get the relevant indices (such as transfer function) and subsequently verified whether there is statistical differences among the results of these five selected data.

After verifying the feasibility of using 2 min RRI series for further analysis, the relevant indices such as gain of transfer function were compared between Normal and ESRD.

\subsubsection{Cross-spectral analysis}

AR model is one of the modern power spectrum estimation methods [10]. AR model equation can be expressed by

$$
x(n)=-\sum_{i=1}^{p} a_{p}(i) x(n-i)+w(n)
$$

where, $w(n)$ is white noise, $p$ is the order of AR model.

The linear interaction of RRI and QTI sequence can be assessed utilizing two-sequence cross-spectral analysis[11]. Bivariate AR process is expressed by

$$
\begin{gathered}
X(n)=-\sum_{i=1}^{p} A_{p}(i) X(n-i)+w(n) \\
\text { where, } X(n)=\left[\begin{array}{l}
\operatorname{rr}(n) \\
\mathrm{qt}(n)
\end{array}\right], W(n)=\left[\begin{array}{l}
W_{\mathrm{rr}}(n) \\
W_{\mathrm{qt}}(n)
\end{array}\right],
\end{gathered}
$$

$$
A(k)=\left[\begin{array}{cc}
a_{\mathrm{rr}}(n) & a_{\mathrm{rr}-\mathrm{q}}(n) \\
a_{\mathrm{qt}-\mathrm{rI}}(n) & a_{\mathrm{qt}}(n)
\end{array}\right] .
$$

The coefficient $a_{\mathrm{rr}-\mathrm{qt}}$ represents the effect of QTI on RRI and $a_{\mathrm{qt}-\mathrm{rr}}$ represents the effect of RRI on QTI, by which the causal relationship of the two series is considered. Parameters in the model are determined by the least square method. The order of the AR model $(p)$ is determined by Akaike information criterion and the maximum value is limited to 16 . After the cross-spectral function is obtained , integral operation is subsequently performed to obtain the total energy of the cross-spectrum in the LF and HF band separately, and the ratio of the two is calculated as LF/HF for both day $\left((\mathrm{LF} / \mathrm{HF})_{\mathrm{d}}\right)$ and night $\left((\mathrm{LF} / \mathrm{HF})_{\mathrm{n}}\right)$, as well as the ratio of them $\left((\mathrm{LF} / \mathrm{HF})_{\mathrm{d}} /(\mathrm{LF} / \mathrm{HF})_{\mathrm{n}}\right)$.

\subsubsection{Coherence function and transfer function}

Coherence function can be used to characterize the strength of the linear coupling of RRI and QTI and is assessed as

$$
r^{2}(f)=\frac{\left|p_{\text {rr-qt }}(f)\right|^{2}}{p_{\text {rr }}(f) p_{\text {qt }}(f)}
$$

where, $p_{\text {rr-qt }}(f)$ is the cross-spectral function of RRI and QTI, $p_{\mathrm{rr}}(f)$ and $p_{\mathrm{qt}}(f)$ are the power spectrum functions of RRI and QTI, respectively. Coherence function ranges from 0 to 1 , indicating a perfect uncorrelation and a full correlation. In contrast to traditional coherence function, the exploitation of $p_{\mathrm{rr}-\mathrm{qt}}(f)$ and $p_{\mathrm{qt}-\mathrm{rr}}(f)$ here can not only reflect the strength of the linear coupling of RRI and QTI, but to tell the causal direction of them two.

The transfer function provides a frequency domain characterization of the linear relationship of the two signals and its estimation is available on condition that the input-output relationship of RRI and QTI is settled. Since the dominant causal direction is from RRI to QTI under the normal state, we only consider the circumstance of RRI as input and QTI as output. And let $p_{\mathrm{qt}-\mathrm{rr}}(f)$ be zero to eliminate the effect of the opposite direction. Thus, the transfer function expression corresponding to this transfer direction is

$$
H(f)=\frac{p_{\mathrm{rr}-\mathrm{qt}}(f)}{p_{\mathrm{rr}}(f)}
$$

In the LF and HF bands, the gain values at the maximum coupling (i.e., at the frequency corresponding to the peak of the coherence function) of the transfer function were taken as representative values $G_{\mathrm{LF}}$ and $G_{\mathrm{HF}}$ separately for both day $\left(G_{\mathrm{LF}-\mathrm{d}}\right.$ and $\left.G_{\mathrm{HF}-\mathrm{d}}\right)$ and night $\left(G_{\mathrm{LF}-\mathrm{n}}\right.$ and $\left.G_{\mathrm{HF}-\mathrm{n}}\right)$, as well as the ratio of them $\left(G_{\mathrm{LF}-\mathrm{d}} / G_{\mathrm{LF}-\mathrm{n}}\right.$ and $\left.G_{\mathrm{HF}-\mathrm{d}} / \mathrm{G}_{\mathrm{HF}-\mathrm{n}}\right)$. 


\subsubsection{Statistic analysis}

A one-way analysis of variance (ANOVA) was applied to test whether the significant differences of the crossspectral indices exist (including both day and night).

For the comparison of diurnal variation of crossspectral indices in Normal and ESRD, a paired $t$ test was performed. If the normality test (Kolmogorov-Smirnov test) was not fulfilled, the 2 related rank sum test (Wilcoxon test) was utilized. And in the case of differences of the cross-spectral indices between Normal and ESRD, we performed an unpaired $t$ test. If the normality test (Kolmogorov-Smirnov test) was not fulfilled, the 2 independent rank sum test (Mann-Whitney $\mathrm{U}$ test) was utilized. Statistical significance was accepted at the level of $P<0.05$.

\section{Results}

Table 1 shows the statistical results of ANOVA for five randomly selected 2 min series. It was revealed that no significant difference exists for all the cross-spectrum based indices among the five different datasets $(P>0.05)$ regardless of day or night. Therefore, we randomly chose one of the five them for analysis.

The results of time-domain and the cross-spectral analysis were reported in Table 2. From the time-domain indices, it could be seen that diurnal variation of RRI and QTI exists in both Normal and ESRD. In addition, there were significant differences of all the time-domain indices between Normal and ESRD except for QTI at night. Specifically, RRI by day in Normal was larger than that in ESRD while the situation is reversed for RRI at night.

With regard to the results of cross-spectral analysis, $G_{\mathrm{LF}}$ and $G_{\mathrm{HF}}$ during daytime were both higher than that at night as well as the LF/HF. For ESRD, no diurnal variation of $G_{\mathrm{LF}}, G_{\mathrm{HF}}$ and $\mathrm{LF} / \mathrm{HF}$ was observed. Besides, in view of the comparison of this two groups, there was significantly increased $G_{\text {LF-d }} / G_{\text {LF-n }}$ in Normal compared with ESRD while $G_{\mathrm{HF}-\mathrm{d}} / G_{\mathrm{HF}-\mathrm{n}}$ was almost at the same level for Normal and ESRD. The (LF/HF $)_{d} /(\mathrm{LF} / \mathrm{HF})_{\mathrm{n}}$ is bigger than 1 for both Normal and ESRD, and the result in ESRD was significantly lower than that in Normal.

Table 1. ANOVA results of the five randomly selected 2-minute series in Normal

\begin{tabular}{lllllll}
\hline \multirow{2}{*}{ Index } & \multicolumn{5}{c}{ Normal $(\boldsymbol{n}=\mathbf{1 8 9})$} & difference \\
\cline { 2 - 5 } & Data(1) & Data(2) & Data(3) & Data(4) & Data(5) & no \\
$G_{\text {LF-d }}$ & $0.65(0.40,0.86)$ & $0.64(0.40,0.84)$ & $0.70(0.45,0.85)$ & $0.67(0.42,0.87)$ & $0.67(0.42,0.87)$ & no \\
$G_{\text {LF-n }}$ & $0.29(0.17,0.50)$ & $0.28(0.15,0.54)$ & $0.31(0.15,0.54)$ & $0.27(0.17,0.49)$ & $0.28(0.14,0.52)$ & no \\
$G_{\text {HF-d }}$ & $0.40(0.24,0.64)$ & $0.40(0.26,0.64)$ & $0.43(0.26,0.66)$ & $0.45(0.29,0.65)$ & $0.42(0.25,0.63)$ & no \\
$G_{\text {HF-n }}$ & $0.27(0.15,0.45)$ & $0.26(0.14,0.49)$ & $0.27(0.15,0.48)$ & $0.27(0.16,0.46)$ & $0.25(0.14,0.49)$ & no \\
(LF/HF) & $1.68(1.08,3.07)$ & $1.85(1.03,3.96)$ & $2.04(1.13,3.17)$ & $2.03(0.94,3.52)$ & $2.08(1.10,3.69)$ & no \\
$($ LF/HF)n & $0.77(0.38,1.59)$ & $0.64(0.33,1.61)$ & $0.71(0.39,1.32)$ & $0.78(0.36,1.65)$ & $0.72(0.33,1.41)$ & \\
\hline
\end{tabular}

Note: Data are expressed as median and Quartile (25th, 75th)

Table 2. Cross-spectral analysis results in Normal and ESRD

\begin{tabular}{|c|c|c|c|c|}
\hline \multirow{2}{*}{ Index } & \multicolumn{2}{|c|}{ Normal $(n=189)$} & \multicolumn{2}{|c|}{ ESRD $(n=43)$} \\
\hline & Day & Night & Day & Night \\
\hline RRI (ms) & $725 \pm 89^{*, \#}$ & $943 \pm 121^{\#}$ & $796 \pm 127^{*}$ & $853 \pm 121$ \\
\hline QTI (ms) & $377 \pm 23^{*}, \#$ & $434 \pm 41$ & $419 \pm 44^{*}$ & $436 \pm 51$ \\
\hline$G_{\mathrm{LF}}$ & $0.67^{*}(0.42,0.87)$ & $0.27(0.15,0.49)$ & $0.56(0.18,0.78)$ & $0.39(0.17,0.78)$ \\
\hline$G_{\mathrm{LF}-\mathrm{d}} / G_{\mathrm{LF}-\mathrm{n}}$ & \multicolumn{2}{|c|}{$2.10^{\#}(1.05,4.00)$} & \multicolumn{2}{|c|}{$1.06(0.70,2.41)$} \\
\hline$G_{\mathrm{HF}}$ & $0.45^{*}(0.29,0.65)$ & $0.27(0.16,0.46)$ & $0.42(0.25,0.73)$ & $0.34(0.19,0.57)$ \\
\hline$G_{\mathrm{HF}-\mathrm{d}} / G_{\mathrm{HF}-\mathrm{n}}$ & \multicolumn{2}{|c|}{$1.47(0.85,3.12)$} & \multicolumn{2}{|c|}{$1.20(0.78,2.11)$} \\
\hline $\mathrm{LF} / \mathrm{HF}$ & $2.03^{*}(0.94,3.52)$ & $0.78(0.36,1.65)$ & $0.73(0.33,1.58)$ & $0.58(0.25,1.45)$ \\
\hline$(\mathrm{LF} / \mathrm{HF})_{\mathrm{d}} /(\mathrm{LF} / \mathrm{HF})_{\mathrm{n}}$ & \multicolumn{2}{|c|}{$2.28^{\#}(1.02,5.49)$} & \multicolumn{2}{|c|}{$1.20(0.56,2.00)$} \\
\hline
\end{tabular}

Note: Data are expressed as mean \pm SD or median and Quartile (25th, 75 th); ${ }^{*} P<0.05$ Day vs Night; ${ }^{\#} P<0.05$ Normal vs ESRD

\section{Discussion}

This study is based on the cross-spectral analysis of RRI and QTI, investigating the changes of ANS behavior in those with high risk for cardiac arrhythmias and sudden cardiac death. On the premise that the reliability of shortterm indices was verified, we proposed to characterize the ANS behavior by comparing the indices of day and night based on the Holter data. The results showed that there were statistical differences for almost all indices when diurnal variation of the calculated results were analyzed in Normal; while few difference was observed in ESRD, indicating the loss of the circadian rhythm. Besides, we also noticed that QTI tend to be extended in ESRD.

The transfer function here (RRI as input, QTI as output) measures the linear dependence of QTI on RRI. 
Reference [6] points out that the dependence relationship is more sensitive to sympathetic activity. The results of this human experiment indicate that dobutamine can increase the fast adaption (i.e , linear correlation of the two signals) of QTI to RRI change. In our study, there was prominent circadian difference in Normal induced by decreased sympathetic activity at night, leading to reduced $G_{\mathrm{LF}}$. As for ESRD, no statistical difference was observed between $G_{\text {LF-d }}$ and $G_{\text {LF-n }}$. The possible reason may be that the overactivity of sympathetic branch still exists while vagus is active at night which results in the deficiency of $G_{\mathrm{LF}}$ reduction and the loss of circadian difference under this pathological condition.

In the contrast analysis of circadian difference characterized by the ratio of day and night, there existed significant differences of $G_{\text {LF-d }} / G_{\text {LF-n }}$ and $(\mathrm{LF} / \mathrm{HF})_{\mathrm{d}} /(\mathrm{LF} / \mathrm{HF})_{\mathrm{n}}$ between Normal and ESRD, while there was no difference for $\mathrm{G}_{\mathrm{HF}-\mathrm{d}} / \mathrm{G}_{\mathrm{HF}-\mathrm{n}}$. Since HF band mainly reflect the influence of vagal tone, the statistical differences between Normal and ESRD described above indicated that the difference between this two groups is primarily caused by the overactivity of sympathetic branch in ESRD. Our research shows that the change in the degree of linear correlation between RRI and QTI carries information about autonomic regulation.

Our research also verified the feasibility of AR model based short-term indices for cross-spectral analysis on directional correlation of RRI and QTI. And the method can be used to process the data in the investigations for short response, such as stress experiment and passive head-up tilt indicated in Ref [12,13]. Moreover, for the purpose of tracking dynamic cardiovascular state, the application of short-term indices might reduce or eliminate non-stationary interference and increase the robustness of results.

\section{Conclusion}

The correlation analysis of RRI and QTI, especially the directional correlation analysis based on transfer function is capable of characterizing physiological and pathological changes of autonomic regulation and provides methodological reference to effective use of time interval series from ECG.

\section{Acknowledgements}

This work received financial support from the National Natural Science Foundation of China through the project 81471746 and 81071225.

\section{References}

[1] Porta A, Bari V, Badilini F, et al. Frequency domain assessment of the coupling strength between ventricular repolarization duration and heart period during graded head-up tilt. Journal of Electrocardiology 2011; 44: 662-8.

[2] Zhu Y, Yang X, Wang Z, et al. An evaluating method for autonomic nerve activity by means of estimating the consistency of heart rate variability and QT variability. IEEE Transactions on Biomedical Engineering 2014; 61: 938-45.

[3] Browne KF, Zipes DP, Heger JJ, et al. Influence of the autonomic nervous system on the Q-T interval in man. The American Journal of Cardiology 1982; 50: 1099-103.

[4] Magnano AR, Holleran S, Ramakrishnan R, et al. Autonomic nervous system influences on QT interval in normal subjects. J Am Coll Cardiol 2002; 39: 1820-6,

[5] Franz MR, Swerdlow CD, Liem LB, et al. Cycle length dependence of human action potential duration in vivo. Effects of single extrastimuli, sudden sustained rate acceleration and deceleration, and different steady state frequencies. J Clin Invest 1988; 82: 972-9.

[6] Seethala S, Shusterman V, Saba S, et al. Effect of $\beta$ adrenergic stimulation on QT interval accommodation. Heart Rhythm 2011; 8: 263-70.

[7] Merri M, Alberti M, Moss AJ.” Dynamics analysis of ventricular repolarisation duration from 24-hour Holter recordings. IEEE Trans Biomed Eng 1993; 40: 1219-25.

[8] Lombardi F, Sandrone G, Porta A, et al. Spectral analysis of short term R-Tapex interval variability during sinus rhythm and fixed atrial rate. European Heart J 1996; 17: 769-78.

[9] Sun ZW, Peng Y. Study on a complex algorithm fordetecting QT interval. Biomedical Engineering and Clinical Medicine 2009; 13: 184-8.

[10] Hu G, Digital Signal Processing. Beijing: Tsinghua University Press, 2003: 528.

[11] Faes L, Widesott L, Greco MD, et al. Causal cross-spectral analysis of heart rate and blood pressure variability for describing the impairment of the cardiovascular control in neutrally mediated Syncope. IEEE Transactions on Biomedical Engineering 2006; 53: 65-73.

[12] Tulppo MP, Kiviniemi AM, Hautala AJ, et al. Physiological background of the loss of fractal heart rate. Circulation 2005; 112: 314-9 .

[13] Porta A, Catai AM, Takahashi ACM, et al. Causal relationships between heart period and systolic arterial pressure during graded head-up tilt. Am J Physiol Regul Integr Comp Physiol 2011; 300: 378-86.

Address for corresponding:

Yi Peng

Department of Biomedical Engineering, Institute of Basic Medical Sciences, CAMS, 5 Dong Dan San Tiao, Beijing 100005, China

pengyi@pumc.edu.cn 